\title{
Changes in sleep patterns during COVID-19 lockdown: correspondence
}

\author{
Rujittika Mungmunpuntipantip ${ }^{1} \cdot$ Viroj Wiwanitkit ${ }^{2,3}$
}

Received: 18 January 2022 / Accepted: 5 February 2022 / Published online: 11 February 2022

(c) The Author(s), under exclusive licence to Japanese Society of Sleep Research 2022

Dear Editor,

We read the article entitled "Changes in sleep patterns of college students in Israel during COVID-19 lockdown, a sleep diaries study" [1]. Lan et al. mentioned that "A lockdown period affects sleep-wake behavior: during lockdown people sleep duration is increased and their sleep onset is delayed. Nevertheless, the circadian preference of individuals is conserved across conditions. [1]." We agree that the effect of COVID-19 lockdown on sleep is interesting. A basic concern on the present study is on the reliability of the sleep diaries study. If there is a good control of bias, sleep diary might be acceptable for assessment of duration but not other parameters [2]. Nevertheless, there might also be an association with the phase of the outbreak in the study setting. When the outbreak period changes, there might be adjustment to the problem and it might affect the characteristics of sleep [3].

\section{Declarations}

Conflict of interest Both authors declare that they have no conflict of interest.
Ethical statement This article does not contain any studies with human participants or animals performed by any of the authors.

Informed consent This type of study formal consent is not required.

\section{References}

1. Lan A, Kotler D, Kronfeld-Schor N, Stukalin Y, Einat H. Changes in sleep patterns of college students in Israel during COVID-19 lockdown, a sleep diaries study. Sleep Biol Rhythms. 2022;10:16. https://doi.org/10.1007/s41105-021-00371-4 (Online ahead of print).

2. Phillips SM, Summerbell C, Ball HL, Hesketh KR, Saxena S, Hillier-Brown FC. The validity, reliability, and feasibility of measurement tools used to assess sleep of pre-school aged children: a systematic rapid review. Front Pediatr. 2021;9:770262.

3. Wang MT, Henry DA, Scanlon CL, Del Toro J, Voltin SE. Adolescent psychosocial adjustment during COVID-19: an intensive longitudinal study. J Clin Child Adolesc Psychol. 2022;10:1-16. https://doi.org/10.1080/15374416.2021.2007487 (Online ahead of print).

Publisher's Note Springer Nature remains neutral with regard to jurisdictional claims in published maps and institutional affiliations.
Rujittika Mungmunpuntipantip rujittika@gmail.com

1 Private Academic Consultant, Bangkok, Thailand

2 Dr DY Patil University, Pune, India

3 Hainan Medical University, Haikou, China 